\title{
BMJ Open Strategies prescribers and pharmacists use to identify and mitigate adverse drug reactions in inpatient and outpatient care: a cognitive task analysis at a US Veterans Affairs Medical Center
}

\author{
Khoa Anh Nguyen (D) , ${ }^{1,2}$ Laura G Militello, ${ }^{3}$ Amanda Ifeachor, ${ }^{4}$ Karen J Arthur, ${ }^{5}$ \\ Peter A Glassman, ${ }^{6}$ Alan J Zillich, ${ }^{7}$ Michael Weiner, ${ }^{1,8}$ Alissa L Russ-Jara ${ }^{1,7}$
}

To cite: Nguyen KA, Militello LG, Ifeachor A, et al. Strategies prescribers and pharmacists use to identify and mitigate adverse drug reactions in inpatient and outpatient care: a cognitive task analysis at a US Veterans Affairs Medical Center. BMJ Open 2022;12:e052401. doi:10.1136/ bmjopen-2021-052401

- Prepublication history and additional supplemental materia for this paper are available online. To view these files, please visit the journal online (http://dx.doi.org/10.1136/ bmjopen-2021-052401)

Received 16 April 2021 Accepted 26 January 2022
Check for updates

(C) Author(s) (or their employer(s)) 2022. Re-use permitted under CC BY-NC. No commercial re-use. See rights and permissions. Published by BMJ.

For numbered affiliations see end of article.

Correspondence to Dr Khoa Anh Nguyen; khoanguyen@cop.ufl.edu

\section{ABSTRACT}

Objective To develop a descriptive model of the cognitive processes used to identify and resolve adverse drug reactions (ADRs) from the perspective of healthcare providers in order to inform future informatics efforts Setting Inpatient and outpatient care at a tertiary care US Veterans Affairs Medical Center.

Participants Physicians, nurse practitioners and pharmacists who report ADRs.

Outcomes Descriptive model and emerging themes from interviews.

Results We conducted critical decision method interviews with 10 physicians and 10 pharmacists. No nurse practitioners submitted ADR incidents. We generated a descriptive model of an ADR decision-making process and analysed emerging themes, categorised into four stages: detection of potential ADR, investigation of the problem's cause, risk/benefit consideration, and plan, action and follow-up. Healthcare professionals (HCPs) relied on several confirmatory or disconfirmatory cues to detect and investigate potential ADRs. Evaluating risks and benefits of related medications played an essential role in HCPs' pursuits of solutions

Conclusions This study provides an illustrative model of how HCPs detect problems and make decisions regarding ADRs. The design of supporting technology for potential ADR problems should align with HCPS' real-world cognitive strategies, to assist fully in detecting and preventing ADRs for patients.

\section{INTRODUCTION}

Adverse drug events (ADEs) harm over 1.5 million patients and cost over $\$ 500$ million each year in USA. ${ }^{1}$ These concerning statistics prompted the US Office of Disease Prevention and Health Promotion to develop a National Action Plan for Adverse Drug Event Prevention in 2014. ${ }^{2}$ This plan called for actions, including developing health information technology to promote best practices in prescribing, and in detecting cases of high risk for ADEs. ${ }^{2}$ Many factors contribute

\section{Strengths and limitations of this study}

- This is the first study to examine the cognitive process that healthcare professionals (HCPS) use to detect and investigate adverse drug reactions (ADRs).

- We used a critical decision method to construct a descriptive model.

- We only interviewed physicians and pharmacists regarding ADRs but not other HCPs such as nurse practitioners, registered nurses or physician assistants.

- We only examined incidents from a single large Veteran Affairs Medical Center and electronic health records system.

to the high incidence of ADEs in the USA, including an increasing number of adverse drug reactions (ADRs), an ageing population and a rising trend in polypharmacy. ${ }^{3}$ ADR, defined as an unintended response to a drug that occurs at normally used doses, is the most common type of ADE. ${ }^{4}$ Healthcare professionals (HCPs) such as physicians and pharmacists are essential in preventing and managing ADRs because they directly prescribe drugs and provide clinical care. However, the processes that individual HCPs adopt to identify, treat and monitor ADRs are not well understood.

Healthcare systems leverage many different approaches to support HCPs in assessing and preventing ADRs, including systematic efforts to collect and review data to assess the risk of ADRs. ${ }^{5-7}$ With advances in the development and implementation of electronic health records (EHRs), alert systems that help HCPs identify and prevent ADEs have become more feasible and promising. Indeed, automated computerised order checks, frequently resulting in drug alerts, 
are often based on triggers such as the documentation of a patient's allergies. ${ }^{8}$

While medication alert systems for allergies have been implemented regularly, alert systems with decision support to identify and resolve newly occurring ADRs are rarely found. In addition, many EHR alert systems have a technology-centric design that fails to take into account the HCPs' cognitive processes and workflow. ${ }^{9-11} \mathrm{HCP}$ decision-centred design, in contrast, focuses on HCPs' cognitive processes and key decisions, such as prescribing a drug, or choosing a drug regimen, as a core function of design. ${ }^{12}{ }^{13}$ With a foundational understanding of the cognitive strategies that HCPs use to prevent and respond to ADRs in clinical practice, healthcare systems and EHR vendors can develop systemic interventions and technologies to help HCPs prevent and resolve ADRs. Recent studies emphasise that cognitive studies are needed to help understand HCPs' decision making ${ }^{914}$ in order to develop more meaningful and effective clinical decision support. Therefore, the objective of this study was to develop a descriptive model of the cognitive processes used to identify and resolve ADRs from the perspective of HCPs in order to inform future informatics efforts.

\section{METHODS}

\section{Study site and participant characteristics}

This research is part of a larger study ${ }^{15} 16$ conducted at a tertiary care Veterans Affairs Medical Center. Eligible HCPs were physicians, nurse practitioners, and pharmacists who prescribed medications, managed medications, or verified prescriptions in an inpatient or outpatient setting. We excluded medical and pharmacy residents, trainees, pharmacy technicians and those with a purely administrative role. We invited all remaining eligible HCPs at the study site to participate. Eligible HCPs were invited to participate via emails, flyers and follow-up via phone calls. All participants consented in written prior to the critical decision method (CDM) interviews. An extended version of the recruitment can be found from the method paper. ${ }^{15}$

\section{Study design}

The study procedure and data analysis are illustrated in four steps in figure 1.

\section{Potential ADR incident capture}

Participants, when encountering a potential ADR, were ask to complete an incident card, ${ }^{15}$ which captured detailed information about the potential ADR incident, such as the type of ADR, suspected medication(s) and when the participant first became aware of the problem. Given that recall is often difficult for tasks and decisions that are made many times each day, ${ }^{17}$ the incident card was designed to help capture an ADR event close to the time of the incident, before it was forgotten or 'merged' in memory with similar events. A similar approach of administering a small set of questions soon after an incident has been shown to increase accuracy of recall days later. ${ }^{18}$ Subsequently, the incident card that HCPs submitted was used to assist with the CDM interviews if the incident was selected, as described below.

\section{Incident selection}

A research team, consisting of a physician, pharmacist and human factors expert, reviewed each incident card to determine eligibility for an interview. Incident cards were reviewed based on five criteria: incident was appropriately addressed, incident required great expertise or consideration, incident had potential to cause serious injury, incident was unique or challenging, or incident would be difficult for trainees to resolve alone.

\section{Critical decision method}

Selected incidents led to scheduling of follow-up CDM interviews with HCPs, to collect further details. ${ }^{15}$ The CDM interview technique, often used as part of a cognitive task analysis, is designed to capture detailed incident accounts from participants, including strategies, critical cues and contextual elements as experienced by the interviewee. ${ }^{17}$ A human factors expert conducted pilot testing of the interview guide with three HCPs (two physicians, one pharmacist) to further refine interview questions prior to data collection. ${ }^{15}$ Interviews were scheduled within 2-4 weeks of the incident date. One human factors scientist, trained in the CDM technique, conducted a 60 min, semistructured CDM interview with each HCP. These interviews were structured in the following three phases:

1. Capture a brief summary of the incident: The interviewer asked the participant to summarise the incident as it pertained to the ADR concern. If multiple eligible incidents were received from a participant, the interviewer asked questions at the beginning of the interview, to select the more challenging incident.

2. Construct an incident timeline: The interviewer asked participants probing questions, to reconstruct a high-

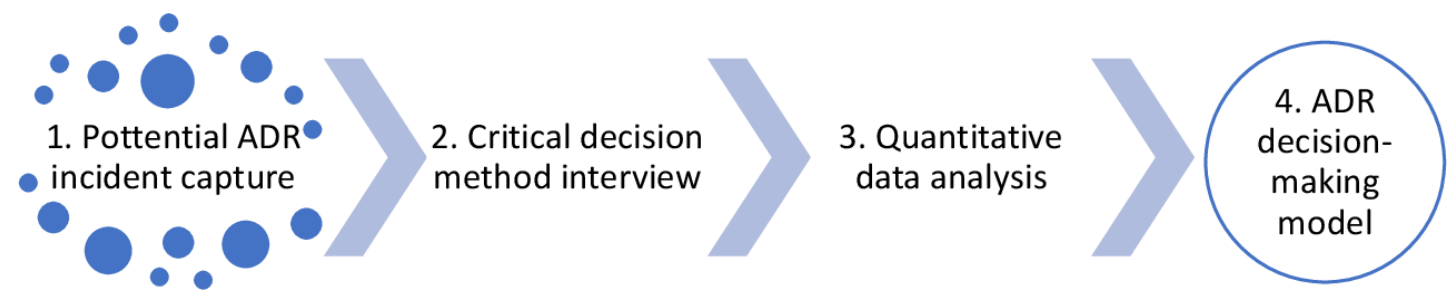

Figure 1 Overview of the study procedure. Four main steps are shown in the illustration. ADR, adverse drug reaction. 
level time line of the incident. This time line was recorded and displayed on a whiteboard, visible to both the interviewer and HCP during the interview session. The time line helped the interviewer organise the incident in chronological order and provided a common point of reference for the rest of the interview.

3. Ask in-depth questions. The interviewer asked additional questions to investigate the HCPs' decision-making process, and to identify cognitive cues used during the course of the incident. The interviewer then asked hypothetical questions to gain further insights about the cognitive cues or strategies that the participant used to detect and respond to the ADR incident.

The incident time line was photographed. Interviews were audio-recorded and transcribed for analysis. The complete interview guide and a more detailed description of study methods is available elsewhere. ${ }^{15}$ Examples of interview questions are below.

1. What caused you to be concerned about the medication for this patient?

2. In what ways did computerised alerts help you notice the right things and take action?

3. What tools/software/technology were used to help manage the medication conflict?

4. What, if any, documentation in the EHR helped you know what to do?

5. Under what circumstances, if any, would you have discontinued the medication for this patient, rather than reducing the dose?

\section{Qualitative data analysis}

Interview transcripts were independently analysed by a human factors engineer and a clinical pharmacist, to generate decision requirement tables for each incident. The decision requirements table is a representation commonly used to examine key information within and across incidents. ${ }^{13}$ Any discrepancies in table content were discussed by the analysts until they reached consensus. ${ }^{19}$ These decision requirements tables summarised each incident and examined key elements. Specifically, researchers identified key decision points inductively, and for each decision point, recorded several components, including cues, strategies, potential errors and any factors that made the decision difficult. (See online supplemental appendix A for a sample decision requirements table.)

Three analysts (human factors engineer, pharmacist researcher and cognitive psychologist) then used these tables to identify initial themes across four selected incidents. Each analyst independently coded the problem detection aspect of each incident from those decision requirements tables, then met weekly to discuss whether the data supported the initial conception of the model, and whether the proposed codes adequately captured the incident. Refinements were made to the codebook, including merging codes and adding new ones, refining the definition of codes, and adding examples to the codebook. When they completed this process for the first four incidents, they worked through the same process with the investigate the cause codes, followed by the codes for plans/ address/follow-up from the decision requirements tables.

After analysing the first four incidents, two of the analysts (pharmacist researcher and cognitive psychologist) independently analysed the remaining data in blocks of four incidents (two pharmacist cases and two physician cases), using the complete codebook. Each incident was discussed until consensus was reached. When a block of four random incidents had been analysed and discussed, the two analysts summarised any new insights and potential refinements to the codebook (ie, clarifying definitions, nesting or un-nesting codes to reflect the data more completely), in consultation with the human factors engineer. See online supplemental appendix B for the final codebook. Final consensus codes were entered into NVivo software (QSR international, V.10), to assist with data management and analysis.

\section{Initial model development of ADR decision-making}

Data from all sources were collected and analysed to develop the initial model of ADR decision-making. To develop the initial model of ADR decision-making, we conducted a card sort of clinicians' key decision points identified in each ADR incident. Decision points from each of the decision requirement tables, such as 'investigate the cause of the problem', were written on individual paper index cards. A multidisciplinary team of five researchers, including two human factors professionals, two pharmacists and one physician, individually sorted cards into meaningful groups based on their background and perspectives to collapse similar decision points across incidents into one decision point, then reviewed and discussed the terminology for each decision point until reaching consensus.

From these card sort results, two human factors professionals developed the initial model, and then reviewed and refined the model monthly with inputs from all research team members, including practising physicians and pharmacists. The model was intended to characterise the cognitive processes that HCPs in our study described as they were confronted with potential ADRs.

\section{Patient and public involvement}

No patient was involved. This study focused on HCPs. However, the results of this study on ADRs were important to improve patient safety in medication use.

\section{RESULTS}

\section{Participants and ADRs reported}

HCPs submitted a total of 35 ADR incident cards. We completed interviews with 10 physicians and 10 pharmacists (table 1) regarding 20 incidents. No nurse practitioners submitted ADR incident cards. Reasons for exclusion of the remaining 15 ADR incident cards include: no action needed for clinical care (1), low ratings from incident cards from reviewers (2), illegible handwriting 
Table 1 Characteristics of participants $(n=20)$ who were Interviewed regarding an ADR incident

\begin{tabular}{lccc}
\hline Characteristic & Physicians $(\mathbf{n = 1 0})$ & Pharmacists $(\mathbf{n}=\mathbf{1 0})$ & All participants $(\mathbf{n = 2 0})$ \\
\hline Gender: female, $\mathrm{n}(\%)$ & $7(70)$ & $6(60)$ & $13(65)$ \\
Age, median, (range) & $42(34-60)$ & $36(29-45)$ & $38.5(29-60)$ \\
Veterans Affairs experience, median, (range) & $10(3.5-26)$ & $7.5(2-13.5)$ & $9(2-26)$ \\
Setting of incident & & & 4 \\
$\quad$ Inpatient & 0 & 4 & 16 \\
$\quad$ Outpatient & 10 & 6 & \\
\hline
\end{tabular}

${ }^{*}$ One pharmacist had both inpatient/outpatient role.

$A D R$, adverse drug reaction.

(1), participant submitted multiple cases (11). Table 2 provides a brief description of the incidents.

\section{ADR decision-making model}

The resulting ADR model (figure 2) outlines participants' cognitive processes when they detected and responded to potential ADRs. This model consists of four medicationrelated stages: problem detection, investigation of the problem's cause, risk/benefit considerations, and plan, action and follow-up. Below, we organise results by the stages in this model. Emergent themes within each stage are underlined, and sample quotes from participants are presented in box 1. Additionally, we describe each of the four stages in the results sections below.

\section{Stage 1. Detection of potential ADR}

In this first stage, HCPs relied on cues that signalled an initial concern. Patient's symptoms (reported) and signs (observed) were common cues to help HCPs recognise a potential ADR. Additionally, abnormal lab values reported within the EHR can also help HCPs detect the problem. Furthermore, HCPs reviewed medication characteristics to sense potential ADRs. Common characteristics of ADR detection were either a new medication that was recently prescribed or started by the patient, or a medication that is commonly known to cause the ADR symptoms that the patient is experiencing. Usual causes of ADR symptoms were often recognised by HCPs from clinical experience. Finally, HCPs sometimes detected a potential ADR based on information (eg, tip-off) from another healthcare professional or the patient themselves.

\section{Stage 2. Investigate the cause of incident}

After detecting the problem, HCPs started to investigate the potential cause. This stage involved information that prompted HCPs to investigate, and their strategies for investigating. For each specific reason to investigate, HCPs used a distinct, corresponding strategy, such as interviewing a patient who reported a certain symptom, or searching in drug references if the HCP suspected that a drug caused the sign or symptom. HCPs also examined confirmatory and disconfirmatory cues, to validate the connection between ADR concerns and their suspected medications.

When investigating the connection between an ADR and a possible problematic medication, HCPs searched for certain confirmatory cues. These were cues that helped providers to conclude that the medication was the likely cause of the ADR. Likewise, HCPs also looked for 'disconfirmatory cues' that suggested that the suspected medication was not causing an ADR (see box 1 for examples of confirmatory and disconfirmatory cues). HCPs often reviewed the disconfirmatory cues through clinical references such as those provided by UpToDate, Micromedex and Lexicomp.

HCPs not only searched for documented and available clinical information but also looked for factors that were absent, to confirm or reject the connection between the ADR concern and the suspected medication. This is known as negative cue. An example includes medications that commonly cause the problem but were not part of the patients' medication regimen.

\section{Stage 3. Risk and benefit considerations}

When the cues indicated the occurrence of an ADR, HCPs often considered the risks and benefits associated with continuing or stopping the suspected medication. As part of this solution-seeking activity, HCPs often considered whether available alternative treatments could alleviate the ADR. They also weighed the risks and benefits associated with such alternatives. While weighing risks and benefits is an essential element of medical decisionmaking, our study found several components that HCPs also take into consideration. These components include:

\section{Severity of side effects}

HCPs often evaluated the severity of the current side effects, and the patient's willingness to tolerate them. The HCP chose to continue the medication, but educated the patient about potential problems, and suggested increased monitoring (eg, follow-up by nursing staff or more frequent patient appointments). In contrast, if the side effects were more severe, the HCP sought alternative medications or treatment.

\section{Anticipated benefits}

If the treatment benefits of the medication were expected to outweigh the risk for the patient, the HCPs tended to continue the medication.

Fostering patient adherence

When investigating options for addressing and solving an ADR, the HCPs also incorporated the patient's feedback 
Table 2 ADR incidents $(n=20)$ selected for interviews

$\begin{array}{llll}\text { Case ID } & \text { Potential ADR incident } & \text { Medication(s) of concern } & \text { Action(s) taken by the } \\ \text { participant }\end{array}$

B.Incidents reported by pharmacists

\begin{tabular}{|c|c|c|c|}
\hline$\# 1$ & Patient reported chest tenderness & Spironolactone & $\begin{array}{l}\text { Switched from spironolactone to } \\
\text { eplerenone }\end{array}$ \\
\hline \#2 & Patient reported nightmares & Varenicline & $\begin{array}{l}\text { Stopped varenicline, prescribed } \\
\text { nicotine patch }\end{array}$ \\
\hline \#4 & Patient reported chest pain & Montelukast & Stopped montelukast \\
\hline \#6 & Patient reported rash & Piperacillin +tazobactam & Switched to clindamycin \\
\hline$\# 7$ & Patient reported chest pain & Abiraterone & $\begin{array}{l}\text { Reaffirmed to the patient that it is } \\
\text { not a heart attack } \\
\text { Continued abiraterone for cancer } \\
\text { treatment }\end{array}$ \\
\hline$\# 10$ & $\begin{array}{l}\text { Electronic health record alerted the } \\
\text { participant about a sulfa allergy }\end{array}$ & Furosemide (new order) & $\begin{array}{l}\text { Advised the nurse practitioner that } \\
\text { furosemide can be started }\end{array}$ \\
\hline
\end{tabular}

ADR, adverse drug reaction; ALT, alamine aminotransferase; AST, aspartate aminotransferase; HCP, healthcare professional; PCP, primary care provider.

and the likelihood that the patient will abandon treatment with the medication in question. Rather than sequentially trying a series of closely related medications (medications in the same class) in hopes that one will have the desired benefits without the adverse reaction, the HCP sometimes switched to a different class of medication therapy to help alleviate the patient's concern.

Which risk is greater?

In some cases, HCPs framed the problem in terms of which risk was greater: stopping the medication, or experiencing the ADR. Conversely, at times they assessed which benefit was greater. This happened most often for patients with particularly challenging health conditions, such as HIV or multiple chronic comorbidities. In these cases, the patient may be taking a medication that has benefits for managing a chronic condition (eg, treating HIV), but stopping the medication may have benefits for mitigating ADR.

\section{Stage 4. Plan, action and follow-up}

The last stage of this decision-making model includes planning, selecting actions and follow-up with patients. 


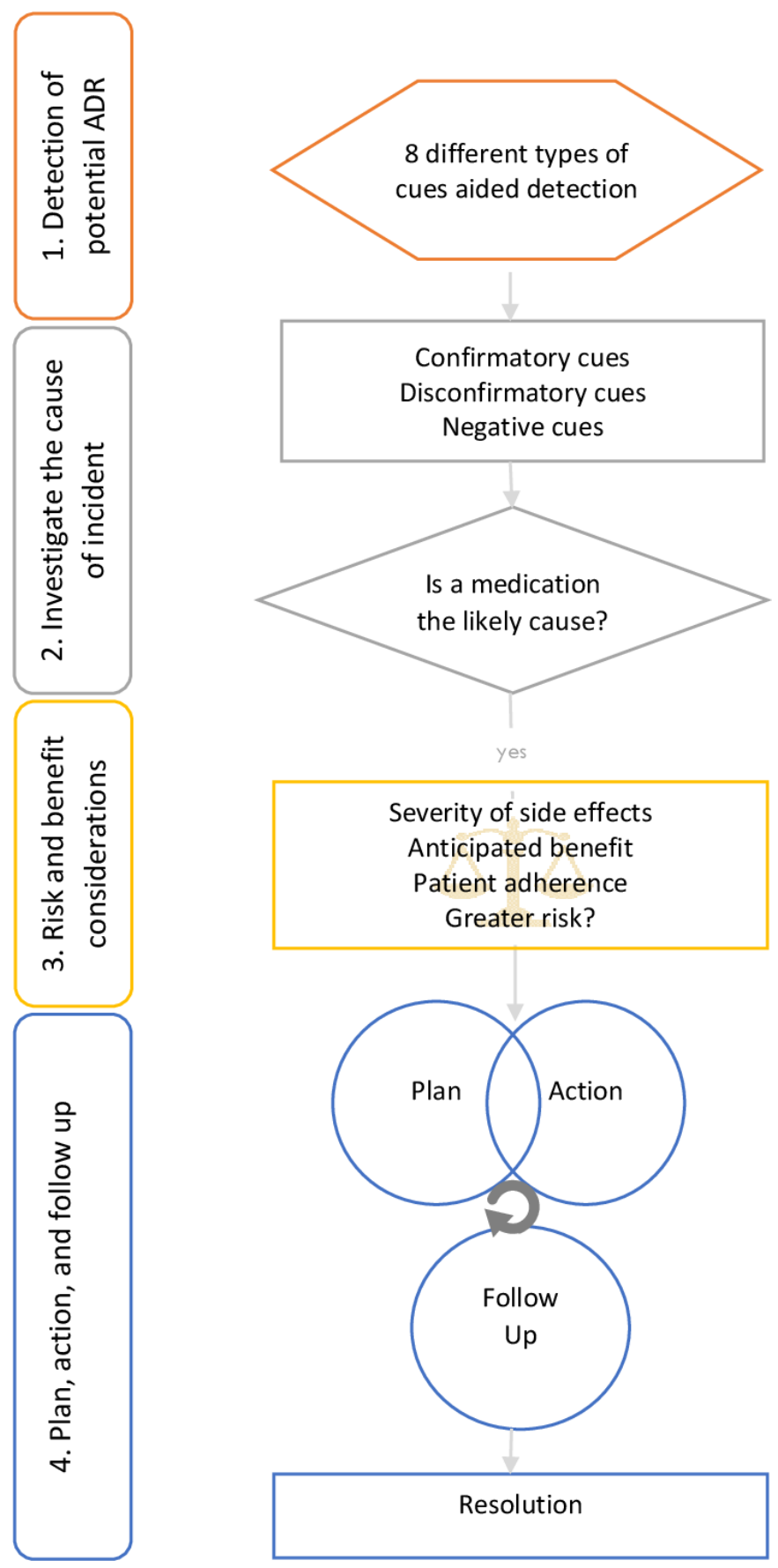

Figure 2 Adverse drug reaction (ADR) decision-making model. the model has four stages.

Based primarily on information collected stages 2 and 3 , HCPs would determine appropriate plans and actions to address the problem. The three main actions in response to incidents in our study included changes to medication management, patient counselling, and follow-up appointments and monitoring. Changes to medications included stopping the offending medication, temporarily placing the medication on hold until the ADR was alleviated, changing the drug to one that is unlikely to cause the ADR or continuing the medication if its perceived benefits outweigh risks.

\section{Box 1 Main themes and quotes from participants}

1.Detection of potential adverse drug reaction (ADR) HCP detected potential ADR based on symptoms and signs Patient called ((the)) pharmacy ((and)) said ((that)) after only 3 capsules of Cymbalta, the patient had muscle spasms and chest muscle spasms. Physician \#1

A new medication or a medication that is already known to commonly cause the ADR symptoms

I asked the patient if he was taking the atorvastatin. Patient ((said)) 'Yes, and (I) have had a lot of diarrheadiarrhoea since starting the medication'. Pharmacist \#8

Information from another healthcare professional (HCP)

I got a view alert ((notification in my EHR inbox)), so I think ((the patient)) called the pharmacy and then they sent me a triage note with a box checked ((for)) adverse reaction. Physician \#1

\section{Investigate the cause of the problem}

Reasons and strategies for investigating

((Participant)) interviewed a patient and asked follow-up questions ((including)): having trouble breathing, sweating? when did the burning sensation start? Pharmacist \#7

\section{Confirmatory cues}

Via phone call, ((the)) patient reported chest pain and muscle spasms. These ((symptoms)) occurred within the first 15 minutes min of taking ((the medication)) ((Cymbalta)). Symptoms consistently happened within 15 min for each of the three doses. ((Confirming that these symptoms were from taking Cymbalta.)) Physician \#1

Disconfirmatory cue

Before starting Cymbalta ((the suspected medication)), the patient had reported muscle spasms/muscle cramps all over as part of a general pain syndrome. Physician \#1

Negative cue (ie, information that is absent)

I rechecked (AST/ALT) on the end of October... and they were ((about)) the same ((as before)). The AST was 204 and ALT was 434.... Since ((it)) was about the same, liver function issues ((were)) NOT due to patient drinking. 'I was hoping maybe ((the cause was)) was drinking ((which is easier to address than an ADR)).' Physician \#7

\section{Risk and benefit considerations}

\section{Severity of side effects}

Patient ((is)) tolerating the pain, not doing anything to compensate. Pain ((is)) not effecting ((sic)) patient's behaviour (in the context of chemotherapy, the potential benefits are very high). Pharmacist \#7

Anticipated benefits

Furosemide ((if taken)) will take fluid off patient's lungs ((patient had sulfa allergy)). Pharmacist \#10

\section{Encouraging patient adherence}

So if you try several ones ((statins)), eventually sometimes patients are like: 'I'm not doing this anymore.' So I didn't want to put him on something that had a decent risk and run the risk that he would have a problem and refuse statins down the line. Pharmacist \#8

\section{Which risk is greater?}

(I need to)) make sure that the risk of anticoagulating her ((with heparin)) was not greater than the risk of having this aortic dissection ((bleeding risk)). Pharmacist \#3

\section{Plan, action and follow-up}

\section{Stop/put on hold/ adjust the medication}

(I) told the patient to stay off Effexor... I wanted to be on the safe side... (I) wanted to make sure it was not an activation syndrome, which I have seen before. Physician \#3. 


\section{Box 1 Continued}

I alerted the patient's primary care provider ((PCP)) and the PCP discontinued the varenicline. Pharmacist \#2

Delayed start of bivalirudin until 3 days days later because patient had had an ablation procedure, ((so I)) felt patient's risk of bleeding was higher. Pharmacist \#3

Patient communication

(I) encouraged ((the)) patient to titrate up on gabapentin, as previously planned... I also try and address issues with diabetic socks/shoes so the patient does not perseverate. I discussed with patient and underscored the need for regular use of capsaicin, ((including)) how it works and why we use it. Physician \#2

Follow-up and monitoring patients

At the patient's next routine nursing visit, ((the)) nurse asked me to meet with the patient again. I briefly examined the patient and he had no signs of an allergic reaction. Physician \#4

\section{Documenting the ADR in the EHR}

(I) entered nightmares for varenicline into the ((EHR)) allergy section. I was adding it to his allergies so no one would reorder it ((again)). Pharmacist \#2

ADR, adverse drug reaction; ALT, alamine aminotransferase; EHR, electronic health record.

Themes (bolded) and quotes (italicised) are organised based on the four stages of the ADR decision making model (figure 2). The numbers for each participant map to the incident descriptions in table 2.

Patient communication was an important activity that HCPs used, in some cases, to initiate shared decisionmaking with the patient about the new medication action and plan. After the new action was taken to mitigate the ADR, the HCP would follow-up and monitor the new action and plan with patients. HCPs used various strategies to monitor and follow-up with patients, such as ordering a new lab test, or examining the patient at the next visit, to ensure that the ADR concern was resolved. Finally, if the ADR was confirmed during the investigation stage, several HCPs reported documenting the ADR in the EHR to help prevent recurrence. HCPs also followed up with other HCPs, to confirm that the ADR was correctly documented in the EHR if they did not input it directly.

It is important to recognise that the decision-making steps in stage 4 , plan, action and follow-up, are iterative steps. If a certain plan and action did not resolve the suspected ADR, HCPs described developing a new plan and action until the problem is resolved.

\section{DISCUSSION}

To our knowledge, this is the first study to examine the cognitive processes of HCPs when they detect and solve potential ADR incidents. Our study explored the iterative steps of HCPs' decision-making, from detection of potential ADR incidents to plans and actions they took to resolve incidents. The study illustrates the cognitive complexity of the work conducted by HCPs when resolving ADR problems. Our findings point to four important implications, discussed below, to develop novel clinical decision support systems that more closely align with HCPs' cognitive workflow.

\section{Develop technologies to facilitate communication between HCPs and patients regarding ADRs}

Detecting potential ADRs requires identifying patients' signs or symptoms that may be related to a drug. ${ }^{20}$ For ADR alerts to work more efficiently, EHR systems could collect reliable information about patients' signs and symptoms, and then display and communicate that information. Therefore, patient-generated data, which is not a typical element of many current alerting systems, may play an important role in future decision support for ADRs. ${ }^{21}$ Additionally, communication between HCPs and patients also plays an important role in resolving ADRs that are already occurring. In some cases, HCPs may help the patient set realistic expectations about the type and severity of symptoms. Throughout the course of ADR investigation and treatment, HCPs need to communicate closely with the patient, to share potential risks and benefits and engage patients in a shared decision-making process. $^{22}$ The study by Topaz et al demonstrated that HCPs were more likely to override an alert for medications that patients are currently tolerating or have previously tolerated. ${ }^{23}$ Indeed, communicating with patients about symptoms, medical history, and so on, is an important element in HCPs' decision-making. Such communication may occur not only through direct patient interactions but also indirectly through patient portals, secure messaging and other telehealth technologies. ${ }^{24}$ These modalities may also increase patients' access to care, facilitating patient-HCP communication to resolve ADRs.

\section{Incorporate confirmatory and disconfirmatory ADR cues into alerting systems}

Rapid, accurate and complete information is important to help detect and resolve ADR problems. Our study found that additional information on ADR detection, such as the confirmatory cues and disconfirmatory cues, were important to assist HCPs with the ADR investigation process. HCPs used these cues to organise and assemble a constellation of information in a form that is meaningful for sensemaking. This concept is known as knowledge-base cognitive mode. ${ }^{25}$ Subsequently, EHR alerts systems for ADRs should align with a knowledgebase cognitive mode by including important cues while filtering distracting information to optimally help HCPs with clinical decision-making. Since ADR alerts detect potential ADRs from symptoms and abnormal lab results, confirmatory and disconfirmatory cues provided by the alerts could allow HCPs to confirm the alerts' trustworthiness and avoid overlooking alerts. In fact, a study on drug allergy alert-overrides showed that providers were more likely to override alerts derived from non-important cues. ${ }^{23}$ Our findings, in combination with those results, highlight the importance of providing cognitive cues to assist HCPs with decision-making. 


\section{Design alert systems that account for negative cues}

The absence of certain cues, known as negative cues, is another important factor for decision-making. HCPs are aware of this absence of information based on familiar perceptual patterns. For example, one experienced provider in our study described how $\mathrm{s} /$ he confirmed that a patient did not have a myocardial infarction (table 2, case B7). Although the patient reported a burning chest sensation, the patient did not have other symptoms such as numb fingers, arm pain or diaphoresis. The absence of the companion symptoms was reassuring, allowing the provider to focus on other potential causes of the symptoms, such as a potential ADR with abiraterone. This type of negative cue is often overlooked in technology-centric design but is a critical component of decision-making, especially during the evaluation of potential ADRs. Complex algorithm development that includes negative cues could advance decision support and help HCPs better detect and investigate potential ADRs.

\section{Advance alert information to better support HCPs' risk-benefit evaluation}

Our ADR decision-making model (figure 2) underscores that even when an ADR is confirmed, HCPs still need to evaluate the medication's risks and benefits. Existing medication alert systems identify potential problems but the information they provide is often insufficient to help HCPs weigh risks and benefits of the problematic medication. As a result, HCPs seek information from drug references, colleagues and other sources. Indeed, ADR alert design without consideration of risks versus benefits is likely to 'fail' (be overridden) in many instances. For complex risk-benefit evaluation, ADR alerts should provide quick access to a comprehensive list of risks and benefits, or corresponding sections of drug information resources, to help HCPs make a sound clinical decision. ${ }^{13}$

This study has several limitations. First, we interviewed only physicians and pharmacists regarding ADRs, since incident submissions from nurse practitioners were low. Nurse practitioners, registered nurses and physician assistants, also interact with patients and play important roles in ADR decision-making, warranting further research. Second, we were not able to directly compare the decision-making processes of physicians and pharmacists, because the variation in incidents precluded direct comparisons (eg, involved different medications or patients with varying medical conditions). Third, we examined incidents from a single large Veteran Affairs Medical Center and EHR system. Our findings might not always apply to other settings. Fourth, all incidents collected and analysed were voluntarily provided by HCPs. This could influence our findings, because HCPs might be more inclined to submit incidents for which they felt most confident in their handling of the ADR; thus, incidents that created even greater challenges or uncertainty for HCPs as they resolved ADRs may have not have been captured. Fifth, most incidents we studied, especially those from physicians, were obtained from outpatient care. Thus, our results might not always apply to inpatient settings. Finally, we were not able to fully incorporate two incidents (box 1, Physician \#8 and Pharmacist \#10) into our decision-making model. Unlike other incidents, these two incidents focused on prevention of an ADR instead of resolving suspected ADRs. Further research is needed on HCPs' decision-making with respect to ADR prevention.

Our study findings have several practical applications. The ADR decision-making model we developed through this research has important implications for the design of clinical decision support. This model could be used as the foundation for a novel ADR alert system to support HCPs in recognising and resolving ADRs. Evolving alert systems by applying this model is anticipated to increase the cognitive support of alerts for HCPs, which subsequently can improve medication safety outcomes for patients. In addition, the ADR decision-making model is expected to be valuable for teaching medical and pharmacy trainees about the decisionmaking process for ADR mitigation.

\section{CONCLUSION}

This is likely the first study to identify the decision-making processes of HCPs when they detect and resolve potential ADR incidents. The ADR decision-making model developed from this study consists of four important stages: problem detection, investigating the cause of the problem, consideration of risks and benefits, and plan, action and follow-up. This model is expected to be valuable for alert system designers as well as educators who are training future physicians and pharmacists about ADR decision-making. Our findings have four key implications for clinical decision support systems: develop technologies to facilitate communication between HCPs and patients to better detect and address ADRs; incorporate confirmatory and disconfirmatory cues into ADR alerting systems to more closely align with cognitive strategies from HCPs; design alert systems that account for negative cues to further facilitate decision making; and enhance alert system displays to better support HCPs' risk-benefit evaluation process for ADRs. The design of supporting technologies for potential ADR problems should align with the real-world cognitive strategies of HCPs in order to achieve the best health outcomes for patients.

\section{Author affiliations}

${ }^{1}$ Center for Health Information and Communication, Richard L Roudebush VA Medical Center, Indianapolis, Indiana, USA

${ }^{2}$ Department of Pharmacotherapy and Translational Research, University of Florida College of Pharmacy, Gainesville, Florida, USA

${ }^{3}$ Applied Decision Science, LLC, Dayton, Ohio, USA

${ }^{4}$ Richard L Roudebush VA Medical Center, Indianapolis, Indiana, USA

${ }^{5}$ VA Health Services Research and Development Center on Implementing EvidenceBased Practice, Roudebush VA Medical Center, Indianapolis, Indiana, USA

${ }^{6}$ Pharmacy Benefits Management Services, VA Greater Los Angeles Healthcare System, Los Angeles, California, USA

${ }^{7}$ Department of Pharmacy Practice, Purdue University, College of Pharmacy, West Lafayette, Indiana, USA

${ }^{8}$ Regenstrief Institute Inc, Indianapolis, Indiana, USA

Acknowledgements The authors thank the study participants for making this work possible. The authors also thank Rachel Dismore, Zamal Franks, Amy Brandt, 
Linda Collins and Steven Sanchez for assistance with procedures, and Rachel Dismore for her assistance with participant recruitment and incident card collection.

Contributors KAN is responsible for the overall content as guarantor. ALR-J wrote the funded grant proposal (VA HSR\&D CDA 11-214), with input from PAG, LGM and MW. All authors (ALR-J, LGM, KJA, PAG, AJZ, MW), except Al and KAN, participated in study planning meetings and contributed to the design of the larger study. LGM trained ALR-J on cognitive task analysis and the critical incident technique. ALR-J conducted all cognitive task analysis interviews. ALR-J and Al analysed interview transcripts to develop a decision requirements table for each incident. KAN and LGM analysed decision requirements tables to identify emergent themes across incidents, under the mentorship of ALR-J, KAN drafted the journal manuscript. All authors reviewed and made important scientific edits to the manuscript and approved it for submission.

Funding This work was supported by VA HSR\&D Career Development Award 11-214 (PI: ALR-J) along with the Centre for Health Information and Communication, Department of Veterans Affairs, Veterans Health Administration, Health Services Research and Development Service, CIN 13-416 (PI: MW). MW is Chief of Health Services Research and Development at the Richard L Roudebush Veterans Affairs Medical Centre in Indianapolis, Indiana, USA. Manuscript writing was supported in part by VA IIR 16-297. KAN was funded in part by the VA Postdoctoral Associated Health Fellowship Programme in Medical Informatics, VA Office of Education, HFP 04-148.

Disclaimer Views expressed in this article are those of the authors and do not necessarily represent the views of the Department of Veterans Affairs or the U.S. government.

Competing interests LGM is co-owner of Applied Decision Science, LLC, a company that studies decision making in complex environments and utilises the critical decision method. She aided in the design of the cognitive task analysis approach used in this study and trained the interviewer. MW has stock in Allscripts and Express Scripts Holding Company. All other authors report they have no conflicts of interest.

\section{Patient consent for publication Not applicable.}

Ethics approval This study involves human participants and was approved by the Indiana University IRB (protocol 1301010433).

Provenance and peer review Not commissioned; externally peer reviewed.

Data availability statement Data are available upon reasonable request. Data from this study will be available upon reasonable request.

Supplemental material This content has been supplied by the author(s). It has not been vetted by BMJ Publishing Group Limited (BMJ) and may not have been peer-reviewed. Any opinions or recommendations discussed are solely those of the author(s) and are not endorsed by BMJ. BMJ disclaims all liability and responsibility arising from any reliance placed on the content. Where the content includes any translated material, BMJ does not warrant the accuracy and reliability of the translations (including but not limited to local regulations, clinical guidelines, terminology, drug names and drug dosages), and is not responsible for any error and/or omissions arising from translation and adaptation or otherwise.

Open access This is an open access article distributed in accordance with the Creative Commons Attribution Non Commercial (CC BY-NC 4.0) license, which permits others to distribute, remix, adapt, build upon this work non-commercially, and license their derivative works on different terms, provided the original work is properly cited, appropriate credit is given, any changes made indicated, and the use is non-commercial. See: http://creativecommons.org/licenses/by-nc/4.0/.

\section{ORCID iD}

Khoa Anh Nguyen http://orcid.org/0000-0002-0096-0293

\section{REFERENCES}

1 Weiss AJ, Elixhauser A. Characteristics of Adverse Drug Events Originating During the Hospital Stay, 2011: Statistical Brief \#164, in Healthcare Cost and Utilization Project (HCUP) Statistical Briefs. Rockville (M.D.): Agency for Healthcare Research and Quality (U.S.), 2006.

2 Harris Y, Hu DJ, Lee C, et al. Advancing medication safety: establishing a national action plan for adverse drug event prevention. Jt Comm J Qual Patient Saf 2015;41:351-60.

3 Schatz SN, Weber RJ, Reactions AD. Adverse drug reactions, 2015. Available: https://www.accp.com/docs/bookstore/psap/2015B2. SampleChapter.pdf [Accessed 1 Jun 2019].

4 Edwards IR, Aronson JK. Adverse drug reactions: definitions, diagnosis, and management. Lancet 2000;356:1255-9.

5 Meyboom RH, Egberts AC, Gribnau FW, et al. Pharmacovigilance in perspective. Drug Saf 1999;21:429-47.

6 Chapman AB, Peterson KS, Alba PR, et al. Detecting adverse drug events with rapidly trained classification models. Drug Saf 2019;42:147-56.

7 Minhaj FS, Rappaport SH, Foster J, et al. Predictors of serious Opioid-Related adverse drug events in hospitalized patients. $J$ Patient Saf 2021;17:e1585-8.

8 Ramsey A, Sheikh A. Innovations in health care delivery: drug allergy. J Allergy Clin Immunol Pract 2019;7:2143-50.

9 Lintern G, Motavalli A. Healthcare information systems: the cognitive challenge. BMC Med Inform Decis Mak 2018;18:3.

10 Russ AL, Zillich AJ, Melton BL, et al. Applying human factors principles to alert design increases efficiency and reduces prescribing errors in a scenario-based simulation. J Am Med Inform Assoc 2014;21:e287-96.

11 Russ AL, Weiner M, Saleem JJ, et al. When 'technically preventable' alerts occur, the design--not the prescriber--has failed. J Am Med Inform Assoc 2012;19:1119.1-1119.

12 Dopp AR, Parisi KE, Munson SA, et al. A glossary of user-centered design strategies for implementation experts. Trans/ Behav Med 2019;9:1057-64.

13 Militelo LG, Klein G. Decision-centered design. The Oxford Handbook of cognitive engineering. Oxford handbooks, 2013: 267-71.

14 Patel VL, Yoskowitz NA, Arocha JF, et al. Cognitive and learning sciences in biomedical and health instructional design: a review with lessons for biomedical informatics education. J Biomed Inform 2009;42:176-97.

15 Russ AL, Militello LG, Glassman PA, et al. Adapting cognitive task analysis to investigate clinical decision making and medication safety incidents. J Patient Saf 2019;15:191-7.

16 Elkhadragy N, Ifeachor AP, Diiulio JB, et al. Medication decisionmaking for patients with renal insufficiency in inpatient and outpatient care at a US Veterans Affairs medical centre: a qualitative, cognitive task analysis. BMJ Open 2019;9:e027439.

17 Crandall B, Klein G, Hoffman R. Working Minds: A Practitioner's Guide to Cognitive Task Analysis, 2006.

18 Geiselman RE, Fisher RP, MacKinnon DP, et al. Eyewitness memory enhancement in the police interview: cognitive retrieval mnemonics versus hypnosis. J Appl Psychol 1985;70:401-12.

19 Ward P, Schraagen JM, Gore J. Incident-Based methods for studying expertise. Oxford University Press, 2019.

20 Liu F, Jagannatha A, Yu $\mathrm{H}$. Towards drug safety surveillance and pharmacovigilance: current progress in detecting medication and adverse drug events from electronic health records. Drug Saf 2019;42:95-7.

21 Islind AS, Lindroth T, Lundin J, et al. Shift in translations: data work with patient-generated health data in clinical practice. Health Informatics J 2019;25:577-86.

22 Barry MJ, Edgman-Levitan S. Shared decision making--pinnacle of patient-centered care. N Engl J Med 2012;366:780-1.

23 Topaz M, Seger DL, Slight SP, et al. Rising drug allergy alert overrides in electronic health records: an observational retrospective study of a decade of experience. J Am Med Inform Assoc 2016;23:601-8.

24 Jungwirth D, Haluza D. Information and communication technology and the future of healthcare: results of a multi-scenario Delphi survey. Health Informatics J 2019;25:161-73.

25 Rasmussen J. Information Processing and Human-Machine Interaction. In: An approach to cognitive engineering. Elsevier Science Inc, 1986. 